\section{MedienPädagogik}

www. medienpaed.com
Zeitschrift für

Theorie und Praxis

der Medienbildung

ISSN 1424-3636

Themenheft Nr. 14: Qualitative Forschung in der Medienpädagogik

\title{
Das Surfgramm als grafische Darstellung von Surfwegen im Internet
}

Martina Schuegraf

\begin{abstract}
Vorbemerkung
Das folgend vorgestellte Instrument zur Analyse und Darstellung von Surfwegen im Internet, die durch ihre Nutzerinnen und Nutzer vollzogen werden, kann und soll nicht als ein technisch komplexes und "objektive» Daten lieferndes Methodeninstrumentarium betrachtet werden. Es ist vielmehr eine Methode, um im Bereich der qualitativen (Internet-)Forschung in einer strukturierenden und fassbaren Weise Handlungsformen im Internet sichtbar und auf diese Weise auswertbar zu machen. Diese Form der Auswertung geht über die reine Interpretation der Transkripttexte von Interviews hinaus, sollte aber damit trianguliert werden.
\end{abstract}

\section{Forschungsdesign und Erhebung}

Jedes Forschungsvorhaben folgt spezifischen Fragen und Problemstellungen, an die sich unterschiedliche Strategien und Vorgehensweisen knüpfen lassen. Dazu gehören zum einen strategische Überlegungen, wie sich das Untersuchungsfeld erschliessen lässt und Kontakte herstellen lassen, und zum anderen methodische Fragen, welche Erhebungsmethoden sich zur Erschliessung des Forschungsfeldes und zur Beantwortung der Forschungsfrage eignen. Dies kann zudem die Modifikation und Anpassung von Methoden an den Untersuchungsgegenstand implizieren, wenn deutlich wird, dass die vorhandenen Erhebungsverfahren nicht in ihrer eigentlichen Anwendung zur Bearbeitung des Forschungsvorhabens geeignet sind. In bestimmten Fällen erfordert es zudem eine "Neuerfindung», die oft in Triangulation ${ }^{1}$ mit «Altbekanntem» eingesetzt wird. Die Untersuchung von Medien bzw. des Umgangs mit Medien und insbesondere mit neuen Medien im weitesten Sinne, kann bzgl. bestimmter Fragestellungen eine solche Methodenschöpfung erforderlich machen.

Der Ausgangspunkt für meine Forschungsarbeit ist, die Bedeutung von Medienkonvergenz für die Mediennutzenden und die daran gekoppelten identitätsstiftenden Aspekte in den Mittelpunkt einer Untersuchung zu rücken, da diese Perspektive zumeist von den Medienproduzierenden ausser Acht gelassen wird. Der Forschungsgegenstand ist das Musikfernsehen und die dazugehörigen Internetseiten. Am Beispiel der Musiksender habe ich den medienkonvergenten Umgang von Jugendlichen und jungen Erwachsenen mit den in Deutschland empfang-

\footnotetext{
Eine umfassende Einführung zur Triangulation liefert Flick 2004, des Weiteren Paus-Haase 2000 für die Medienrezeptionsforschung mit Kindern. Eine Kritik an gängigen Triangulationsvorstellungen entwickelt Marotzki 1995, S.75ff.
} 
baren Musikkanälen und den dazugehörigen Internetseiten untersucht. Gerade in diesem Kontext ist der Alltagsbezug von grosser Bedeutung, um die Praktiken der Jugendlichen und jungen Erwachsenen in ihrem Lebenszusammenhang zu begreifen.

Der Forschungsgegenstand Musiksender eröffnet dabei insbesondere drei Blickrichtungen: Zum einen steht hier der gesamte Sender als Marke mit seinen Musikund Videoclippräsentationen im Mittelpunkt, zum zweiten nutzen insbesondere jüngere und damit zumeist medienaffinere Zuschauerinnen und Zuschauer diese Sender und zum dritten denke ich, dass die Musiksender mit ihren Internetauftritten ein hohes identitätsstiftendes Potenzial bereitstellen. Zudem bietet das Musikfernsehen seinem Publikum Einwirkmöglichkeiten auf seine Sendungsinhalte wie kein anderer Fernsehsender. Die Sendungen dieser Kanäle bauen zum Teil auf die Teilhabe ihrer Zuschauerschaft an den Shows auf. Dies betrifft z. B. das Voten von Musiktiteln, die in bestimmten Sendungen wie den Chartshows, in interaktiven Sendungen wie MTVselect oder in einer bestimmten Zeitschiene wie bei Get the Clip auf VIVAplus gespielt werden.

Für die qualitative Untersuchung führte ich 12 Interviews mit Jugendlichen und jungen Erwachsenen im Alter von 16 bis 24 Jahren im Frühjahr 2003 und 2004 durch. Zur Durchführung der Interviews stellte sich jedoch die Frage nach einer geeigneten Erhebungsmethode. Um an die Relevanzsysteme der Befragten und die Muster der fallspezifischen Strukturierungs- und Handlungsweisen zu kommen, musste eine grösstmögliche Offenheit bei der Interviewführung gewahrt werden, wobei in der qualitativen Sozialforschung von dem Prinzip der Offenheit und dem Prinzip der Kommunikation gesprochen wird (vgl. Marotzki 1995, 62; Bohnsack 2000: 23; Fabel-Lamla/Tiefel 2003, S. 191). Denn im Sinne einer rekonstruktiven Methodologie sollte sich die Interviewmethodik an den Erzählsträngen und Strukturierungsvorgaben der Interviewten orientieren. Durch das Einhalten eines offenen Interviewverfahrens und einer grösstmöglichen Zurückhaltung meinerseits im Interviewkontext wurde den Interviewten die Möglichkeit gegeben, ihre eigenen Relevanzsetzungen vorzunehmen, in ihrer Sprache zu sprechen und ihre Themen zu exponieren, die gegebenenfalls über die von mir antizipierten Themenbereiche hinausreichten.

Für einen umfassenden Einblick in das Vorgehen der Interviewten im Netz war es von Bedeutung, einen Eindruck von ihrem Surfverhalten zu bekommen, um auf dieser Grundlage ihren gewöhnlichen Surfweg rekonstruieren zu können. Daher entschied ich mich nach einem ersten Probeinterview, mit den Jugendlichen und jungen Erwachsenen des Samples im Interview online zu gehen. Das bedeutete jedoch gleichzeitig, dass die Interviews bei ihnen zu Hause durchgeführt werden mussten. Denn nur dadurch ist es möglich, ihre Vorgehensweisen in Korrespondenz zu den Zugangsmöglichkeiten wie technische Voraussetzungen, Voreinstellungen ihrer Computer, Ausrüstung, Stellung des Computers in der Familie etc. zu 
erhalten. Schliesslich interessierte mich ausserdem die Bedeutung der Medien im Allgemeinen, im Tagesverlauf der Interviewten sowie in ihrer biografischen Entwicklung.

Letztlich verknüpfte ich verschiedene Erhebungsmethoden, um meinem Forschungsgegenstand und meiner Fragestellung gerecht zu werden. Zum einen arbeitete ich mit thematisch fokussierten Interviews, angelehnt an Merton/Kendall (1979), die sich an den für meine Forschungsfrage relevanten Themen orientierten. Diese verknüpfte ich mit einer erzählgenerierenden Eingangsfrage, die in der Biografieforschung als Erzählstimulus für die biografische Erzählung verwandt wird (vgl. Schütze 1983, Marotzki 1995, Fischer-Rosenthal/Rosenthal 1997). Diesen Stimulus zog ich als Erzählaufforderung für die letzte Rezeptionssituation von Musikfernsehen heran, denn mit einer Erzählaufforderung, die auf Phänomene zurückgreift, die nicht sehr lange zurückliegen und zu der alle meine Interviewpartnerinnen und -partner etwas berichten können, konnten Vorbehalte der Jugendlichen und jungen Erwachsenen gegenüber der Interviewsituation abgebaut werden. Zudem ermutigte es sie, weitere Narrationen und Deskriptionen anzuschliessen. Darüber hinaus diente das gemeinsame Surfen im Internet einerseits dazu, die favorisierten Musiksenderseiten zu besuchen, andererseits war es wichtig, auch auf jene Webauftritte zu gehen, die zu ihrem sonstigen gewohnheitsmässigen Surfen gehören, um auf diese Weise ihre konkreten Vorgehensweisen im Netz rekonstruieren zu können.

Der Onlinepart wurde von der Methode des lauten Denkens² begleitet. Mittels dieser Methode erzählten sie mir, was ihnen zu den einzelnen Seiten durch den Kopf ging und wie sie die Internetpräsenzen wahrnahmen. Auf dieser triangulierten Basis erarbeitete ich ein methodisches Vorgehen, das die oben vorgestellten Instrumente in Anpassung an mein Forschungsdesign integrierte, und entwickelte einen entsprechenden Leitfaden mit Themenkomplexen für die Interviewdurchführung.

Insgesamt war die Interviewdurchführung dreigeteilt: Der Einstieg erfolgte über das Musikfernsehen. Als erstes befragte ich die Interviewpartnerinnen und -partner zu ihren Musikfernsehgewohnheiten und Musikvorlieben. Meistens ging es in einem zweiten Schritt um die allgemeine Mediennutzung, wobei weitere Medien im Tagesverlauf und in der biographischen Entwicklung zur Sprache kamen. Dies konnte jedoch je nach Verlauf des Interviews variieren und erst nach dem Onlineteil erfolgen. Im dritten Teil wurden die Bedeutung und die konkrete Nutzung des Internets exploriert, wobei die Methode des lauten Denkens zum Einsatz kam. Dieser Interviewabschnitt schloss mit dem Surfen im Internet am häuslichen Rechner.

2 Ein Beispiel zum Einsatz dieser Methode bei der Fernsehnutzung von Jugendlichen beschreiben Bilandzic und Trapp 2000. 


\section{Strategien der Auswertung}

Zur Auswertung der erhobenen Daten gehört die Suche nach einem entsprechenden und geeigneten Instrumentarium. Für die Analyse der Interviewtranskripte fiel die Wahl auf die Grounded Theory, um mittels eines rekonstruktiven Verfahrens die Aussagen und Ausführungen der Interviewpartnerinnen und -partner angemessen erfassen und reflektieren zu können. Als Methodologie ermöglicht ein Vorgehen mit der Grounded Theory nicht nur qualitative Daten zu beschreiben und zu interpretieren, sondern auch theoriegenerierend zu verstehen und zu deuten. Jedoch stellte sich zudem die Frage nach der Auswertung des Onlineteils der Interviews. Hierfür entwickelte ich ein Instrument, das die Vorgehensweisen meiner Interviewpartnerinnen und -partner visualisiert. Dabei sollten die konkreten Vorgehensweisen der Jugendlichen und jungen Erwachsenen im Netz nachvollzogen werden können.

Bevor ich folgend auf die konkreten Auswertungsstrategien bzgl. des Internetteils eingehen werde, möchte ich kurz den theoretischen Hintergrund erläutern, vor dem sich Internetforschung auch als Online-Ethnographie verstehen lässt, um den Rahmen für meine späteren Überlegungen zu entfalten. An dieser Stelle soll angemerkt werden, dass es hier nicht darum geht, einen Überblick über nationale oder auch internationale Ansätze zur Internet- bzw. Online-Forschung (in Bezug auf Jungendkulturen) zu geben. Die sich anschliessenden Ausführungen sollen vielmehr als Rahmen für meine Untersuchung verstanden werden, vor deren Hintergrund die Analyse der Surfgramme stattgefunden hat. Sie erheben auch nicht den Anspruch, in Gänze auf die Auswertung mit dem Surfgramm im Onlineteil anwendbar zu sein, denn viele Erkenntnisse erfolgten unter Hinzunahme der Transkripte.

Internet- bzw. Online-Forschung als Online-Ethnographie zu betrachten, ist das Anliegen Winfried Marotzkis mit einer Untersuchung von virtuellen Communitys, die an der Universität Magdeburg durchgeführt wurde und in der acht verschiedene «Strukturmerkmale» herausdestilliert wurden (vgl. Marotzki 2003, S.155ff.). Das Internet kann nach Marotzki als «kultureller Raum» (Marotzki 2000, S. 245) bzw. als «neuer Kulturraum» (Marotzki 2003, S. 149) verstanden werden. Er definiert Kultur demzufolge als «das jeweils selbstgesponnene Bedeutungsgewebe, in dem Menschen sich selbst entwerfen, ihre Handlungen koordinieren und sich über Prozesse der Symbolisierung, Ritualisierung, Metaphorisierung und Allegorisierung konstituieren» (Marotzki 2000, S. 245).

Zur Differenzierung und Systematisierung der Internetforschung unterscheidet Marotzki erst einmal drei "grundlegende Forschungsfoki» (Marotzki 2003, S. 151). Einen Forschungsfokus sieht er in «Offline»:

Zum einen können Nutzer des Internets und ihre Lebenswelten offline untersucht werden: Wie sie mit dem Medium umgehen und wie sie es in ihre Lebenswelten integrieren, kann Gegenstand der Forschung sein. [...] Der 
Mediengebrauch im Kontext der alltäglichen Lebenswelt steht hier also im Zentrum der Aufmerksamkeit. (Marotzki 2003, S. 151)

Diese Perspektive auf die Mediennutzenden beschreibt auch einen Interessenschwerpunkt in meiner Untersuchung. Mittels der Interviews soll auf diese Weise ein Verständnis für die Verwendungsweisen des Internets im lebensweltlichen Kontext erfasst werden. Ein zweiter Forschungsfokus meint das Verhältnis «OnlineOffline»:

Zweitens kann das Verhältnis von Online und Offline untersucht werden, d. h. es wird untersucht, was von Einzelnen und/oder Gruppen Online an Aktivitäten entfaltet wird und wie diese im Verhältnis zu ihrer Lebenswelt stehen. (Marotzki 2003, S. 151)

Auch dieses Verhältnis lässt sich in meinen Interviews erkennen und aus dem vorhandenen Material rekonstruieren. Es nimmt einen besonderen Stellenwert bei der Interaktion und Kommunikation mittels und durch Medien meiner Interviewpartnerinnen und -partner ein. Der dritte Forschungsfokus wird von Marotzki als Forschungsfokus "Online» (ebd., S. 152) bezeichnet. Hier unterscheidet er zwischen Methoden klassischer Marktforschung wie beispielsweise Logfile-Analysen, User-Tracking und Collaborative Filtering (vgl. ebd.) und qualitativen Methoden wie die Online-Ethnographie, die er insbesondere zur Erforschung virtueller Communitys für aussichtsreich erachtet (vgl. ebd., S. 153). Im Folgenden soll ein knapper Einblick in die Ergebnisse dieser online-ethnographischen Untersuchung von virtuellen Communitys gegeben werden, da sich einige dieser Merkmalsbereiche als heuristisches Mittel zur Rekonstruktion der Surfwege und der Vorgehensweisen sowie Inszenierungen der Interviewten im und mit dem Netz erwiesen. Auch wenn es sich nicht im engeren Sinne um virtuelle Communitys handelt wie beispielsweise Funama (www.funama.de) oder Kidsville (www.kidsville.de), so pflegen zum einen dennoch die Sender einen Austausch mit ihrer "Community», als welche sie ihre Userschaft der Kommunikationsbereiche betrachten. Zum anderen zeigten sich auch Community-Bildungen der Befragten mit anderen aufgrund gleicher Interessenslagen und Aktionismen. Allerdings beziehe ich die folgenden Strukturmerkmale nicht nur auf solche Communitys, sondern auf alle relevanten Websites, die meine Interviewpartnerinnen und -partner nutzen.

Die ersten drei von Marotzki benannten Strukturmerkmale sind für meine Untersuchung nicht von Bedeutung. Das ist zum einen die «Leitmetapher für die Infrastruktur», die sich durch das Aussehen und Auftreten der Community im Netz zeige, z.B. als Stadt wie bei Funama (vgl. ebd., S.156) oder Kidsville. Auch das «Regelwerk» (z.B. Zugangsregelungen, Gratifikationssystem, Sanktionssystem) (vgl. ebd.) und die «Soziographische Struktur» (Positionen durch Kompetenzen, 
Anerkennung, Pflichten etc.) (vgl. ebd., S. 157) sind nicht von weiterem Interesse, obwohl diese teilweise auch in den Kommunikationsbereichen von MTV und VIVA existieren. Die folgenden Merkmale sind allerdings auch für meinen Untersuchungsbereich von Bedeutung, wobei ich diese jedoch immer auf die Perspektive der Nutzung anwende. Erstens ist das die «Kommunikationsstruktur»: Welche Möglichkeiten wie Chats, E-Mail, Newsletter, Foren, ICQ etc. werden bereitgestellt (vgl. ebd., S. 158) bzw. von meinen Interviewpartnerinnen und -partnern genutzt? Zweitens ist das die «Informationsstruktur»: Welche Informationen werden zur Verfügung gestellt (vgl. ebd., S. 159) bzw. genutzt? Drittens beschreibt Marotzki eine «Präsentationsstruktur»: Diese umfasst das Identitätsmanagement mit Nickname und ID-Card oder mit der Erstellung einer eigenen Homepage und zudem die Nutzung halböffentlicher und privater Arenen (vgl. ebd., S. 159ff.). In diesem Zusammenhang interessieren mich zwei Fragen: Wie präsentieren sich die Userinnen und User im Netz (anderen gegenüber) und wie präsentieren sie sich mir gegenüber in der Interviewsituation? Als Viertes geht es um die «Partizipationsstruktur»: Wie werden die Mitbestimmung und die Mitgestaltungsmöglichkeiten geregelt (ebd., S. 161)? Dieses Merkmal zeigte sich als sehr zentral für meine Forschungsfrage, wenn es darum geht, welche Potenziale sich die Mediennutzenden neben den von den Musiksendern angebotenen, wie beispielsweise das Musiktitelvoting, eröffnen. Letztlich spielte auch das «Verhältnis Online-Offline», bei Marotzki das achte Strukturmerkmal (vgl. ebd., S. 162), eine Rolle. Hier geht es um die «Rückbindung an die Alltagswelt der Nutzer» (ebd.). Auch das lässt sich in meinen Interviews finden.

Eine solche online-ethnographische Perspektive auf den Untersuchungsgegenstand Internet (Forschungsfokus Online), in dieser Untersuchung mit der Verknüpfung und dem Schwerpunkt auf der Erforschung der konkreten Umgangsformen der Nutzenden (Forschungsfokus Offline), erweitert m. E. die Sensibilität für das Medium bei einer qualitativen Forschungsanlage. Allerdings ist dabei nicht davon auszugehen, dass sich jedes dieser relevanten Merkmalsbereiche in jedem der Fälle aufzeigen lässt. Durch die Rekonstruktion lassen sich jedoch unterschiedliche Gewichtungen erkennen.

Um schliesslich dem Anspruch der Nachvollziehbarkeit der konkreten Vorgehensweisen der Interviewten im Netz gerecht zu werden, stellte sich die Frage, wie sich eine angemessene Rekonstruktion in geeigneter Weise durchführen lässt. Mittels der Methode des lauten Denkens liegen zum einen die Anmerkungen, Narrationen und Deskriptionen zu den besuchten Websites vor. Diese wurden - wie auch die anderen Teile des Interviews - transkribiert. Hierdurch lassen sich erkenntnisreiche Schlüsse über die Hintergründe bzw. Beweggründe und die Bedeutungen der Vorgehensweisen rekonstruieren. Doch mein Erkenntnisinteresse lag ebenso auf der «visuellen Gestalt» des Surfweges im Netz, also wie bewegen sich die Userinnen und User von Internetseite zu Internetseite und wie gehen sie dabei konkret 
vor. Es ist ein Unterschied - und dies zeigte sich deutlich in der Interpretationsarbeit - ob Sprachliches als Transkript in Textform oder Bildliches (Screenshots, Druckbilder) zur Visualisierung der «Tätigkeit im Netz» zur Auswertung vorliegt. Damit stellte sich jedoch die methodische Frage zur Form der Visualisierung der einzelnen Surfwege.

Die Antwort darauf ist eine zweifache Transformation oder auch doppelte Rekonstruktion des Surfweges:

1) Auf der Grundlage der transkribierten Interviews markierte ich im Onlineteil jede Internetseite, die während des Interviews besucht wurde. Die Information zu den Internetadressen erhielt ich mittels der Methode des lauten Denkens, weil dadurch jegliche Seite angesagt und somit auf Band aufgenommen wurde. Ausserdem notierte ich mir einen Grossteil der Internetadressen während des Interviews. Aus diesen Angaben ergab sich eine chronologische Abfolge der aufgerufenen Internetseiten, was mir ermöglichte, den Surfweg meiner Interviewpartnerinnen und -partner selbst durchzuführen. Von jeder Seite, die ich auf diese Weise im Internet besuchte, erstellte ich ein Screenshot (Druckbild). Die Gesamtkomposition aller Screenshots aus einem Interview ergab somit den Surfweg der jeweiligen Nutzerin bzw. des jeweiligen Nutzers.

Dies ist der erste Transformationsschritt bei der Rekonstruktion.

2) Da es mir jedoch nicht um die Ästhetik bzw. das Abgebildete auf den Screenshots ging (also keine Produktanalyse), sondern um den Weg, den die Userinnen und User genommen haben, sollte dies in einer grafischen Darstellung zum Ausdruck kommen. Hierzu druckte ich sämtliche Screenshots eines Surfweges (einer/s Interviewten) aus und legte sie der chronologischen Abfolge entsprechend aneinander. Dies ermöglichte zum einen Erkenntnisse über das spezielle Vorgehen der/des einzelnen und zum anderen, im direkten Vergleich mit anderen Surfwegen, Erkenntnisse über Gemeinsamkeiten und Abweichungen im Vorgehen. Diese Visualisierungen der einzelnen Surfwege bildeten wiederum den Ausgangspunkt für die Übersetzung in eine geeignete Präsentationsform für die schriftliche Darstellung. Der zweite Rekonstruktionsschritt besteht somit in dem Nachzeichnen der chronologischen Abfolge der besuchten Seiten auf der Basis der Internetadressen, die je nach Websitezugehörigkeit farblich markiert wurden.

Das Ergebnis dieser zweifachen Transformation oder der doppelten Rekonstruktion zur Visualisierung des Surfweges habe ich schliesslich Surfgramm genannt. Das Surfgramm ermöglicht somit die visuelle Darstellung der Surfwege und zeigt auf diese Weise falltypische Charakteristika. Auch wenn die Verlinkungsstrukturen vielfältige, fast unermessliche Möglichkeiten des Netzgebrauchs zulassen oder sogar provozieren, zeigt sich dennoch eine klare Abfolge im Nacheinander angeklickter Links, aufgesuchter Sites und verwendeter Kommunikationsplattformen. 
In der Triangulation mit den Interviewtranskripten lassen sich mittels der Surfgramme Erkenntnisse auf mehreren Ebenen der Nutzung gewinnen: ${ }^{3}$

Erstens wird die Bedeutung des Einstiegs zu Beginn einer Onlinesitzung deutlich. Durch die Rekonstruktion des Netzweges zeigte sich, dass der Anfang eines Internetbesuchs nicht beliebig zu sein scheint. A) kann der Anfang durch die Hervorhebung dessen gekennzeichnet sein, was für die Nutzenden von bestimmter Bedeutung ist. Dies können besonders beliebte wie auch ungeliebte Seiten sein, mit denen sich die Interviewten zur Zeit des Interviews besonders beschäftigen und an denen sie sich abarbeiten. B) kann der Beginn einer Sitzung aus dem Befolgen von Gewohnheiten und Routinen bestehen, wie beispielsweise das Abrufen von E-Mails oder der Wettervorhersage für den kommenden Tag.

Zweitens lassen sich Aussagen über die lineare Bewegung im Internet treffen. Der Netzweg ist entweder die Bewegung hin zu dem, was von Bedeutung bzw. mit einem besonderen Interesse verbunden ist und somit zum Ausdruck kommen soll. Es ist also ein Herantasten an das «Eigentliche». Oder es ist der umgekehrte Weg, die Bewegung zu dem, was nicht täglich genutzt wird und nur von peripherem Interesse ist, also das Aufsuchen von «Randphänomenen».

Drittens zeigen die Vorgehensweisen im Netz verschiedene Handlungsdimensionen, die sich insbesondere in der Triangulation mit den Interviewtranskripten und

\section{Aktivitätsgrad}

Wie und auf welche Weise ist man im Internet aktiv?

- Hohe/schnelle Aktivität vs. längeres Verweilen

- Treiben lassen/Durchsurfen vs. Auswahl treffen/Zielgerichtetheit

\section{Nutzungsgrad}

\section{Welche Angebote werden wie genutzt?}

- Sich vom Angebot inspirieren und lenken lassen vs. Sich am eigenen Wissen orientieren

- Selbstbestimmt durch Routinen, Gewohnheiten, sich auskennen vs. fremdbestimmt durch Verlinkungsstrukturen, durch die Interviewsituation, durch Aufforderungen im Netz

\section{Navigationsgrad}

\section{Wie wird das Internet genutzt?}

- Rein lineares Vorgehen von einer Seite zur nächsten

- Linearität gekoppelt mit Sprunghaftem: Vor- und Zurückgehen auf bereits besuchte Seiten

\section{Konvergenzgrad}

Welche Seiten werden zu einem konkreten, interessierenden Gegenstand/Phänomen herangezogen?

- Orientierung an Hinweisen aus anderen Medien, Aufmachern, Verweisen vs. Einbringen eigener Kenntnisse über Informationen zu Angeboten auf verschiedenen medialen Plattformen

Abbildung 1: Handlungsdimensionen im Internet

3 Somit war mein Forschungsdesign von Triangulationen auf zwei Ebenen gekennzeichnet: zum einen von einer «methodeninternen Triangulation» (Flick 2004, S. 27), bei der ich verschiedene Erhebungsmethoden zu einem Interviewinstrumentarium verknüpfte, um dadurch unterschiedliche Zugänge zum Forschungsgegenstand zu gewinnen. Zum zweiten von einer Datentriangulation (bei Flick «Triangulation von Datensorten» [ebd., S.36]), bei der ich die Interviewtranskripte in Korrespondenz zu den Surfgrammen stellte und auswertete, um hierdurch verschiedene Perspektiven an den Forschungsgegenstand herantragen zu können. 
den Aufzeichnungen während und nach den Interviews verdeutlichen lassen. Es sind 4 Handlungsdimensionen, die sich hier herauskristallisierten:

In Bezug auf den Aktivitätsgrad geht es um die Frage, wie und auf welche Weise die Nutzerinnen und Nutzer im Internet aktiv sind (schnelle/hohe Aktivität vs. längeres Verweilen, Treiben lassen vs. Zielgerichtet sein). Der Navigationsgrad versucht die Frage zu klären, wie bzw. in welcher Form das Internet genutzt wird. Zeigt sich ein deutlich lineares Vorgehen, also eine klare Struktur oder ist der Umgang mit dem Medium durch Sprunghaftigkeit und ein ausgeprägtes Vor- und Zurückgehen auf bereits besuchte Seiten gekennzeichnet. Der Nutzungsgrad soll die Frage beantworten, welche Angebote werden überhaupt genutzt. Lässt man sich von Angeboten im Netz inspirieren und lenken oder orientiert man sich beim Vorgehen eher an eigenen Wissen und Erfahrungen (selbstbestimmt durch Routinen, Gewohnheiten, Wissen oder fremdbestimmt durch Verlinkungsstrukturen, die Interviewsituation). Als vierten Punkt spielt der Konvergenzgrad insbesondere für meine Untersuchung eine grosse Rolle. Hier geht es um die Frage, welche Seiten zu einem konkreten und interessierenden Gegenstand bzw. Phänomen herangezogen werden (Orientierung an Hinweisen aus anderen Medien, anderen Angeboten oder Einbringen von eigenen Kenntnissen über Informationen zu Angeboten und Erfahrungen im Netz).

Bei diesen Handlungsdimensionen handelt es sich um vier gleichgewichtige Gebrauchsformen des Internets, die bei der Frage nach dem konkreten Umgang mit medienkonvergenten Angeboten emergiert sind. Diese verschiedenen Formen werden jeweils durch ein bipolares Spannungsfeld markiert, wobei die Pole lediglich als (End-)Marken des Feldes begriffen werden können. Diese vier Formen haben die Funktion, den Blick auf die konkreten Vorgehensweisen der Interviewten im Internet bei der Rekonstruktion zu sensibilisieren und damit zu schärfen. Denn mittels dieser Differenzierung werden Nuancierungen der Gebrauchsweisen deutlicher.

Der wissenschaftliche Mehrwert der Surfgramme liegt letztlich in dem Sichtbarmachen von möglichen Deutungen bzgl. der Surfwege. Durch die Arbeit mit den Interviewtranskripten lassen sich zwar die verschiedenen Webseiten, die während des Interviews aufgesucht werden, herauskristallisieren, doch bleiben die Abfolge und die Linearität des Weges unscharf. Denn durch die Erläuterungen und Ausführungen zu den einzelnen Seiten im Interview verwischen die klaren Zusammenhänge zwischen den einzelnen Seiten, die letztlich den Surfweg ausmachen, so dass sich Surfstrukturen nicht einfach herausschälen lassen. Dieses Gebilde erhält man erst durch die visuelle Gestalt in Form der aneinander gereihten Screenshots, wodurch quasi "gestalthaft» typische Vorgehensweisen im Netz ins Auge springen. Und noch deutlicher wird dies im Vergleich der Surfwege. Die textbasierten Interviews können in keiner Form die falltypischen Vorgehensweisen im Vergleich 
der Fälle ähnlich eindrucksvoll verdeutlichen wie die visuelle Darstellung der Surfwege. Um in einem nächsten Schritt dieses konkrete, erfasste Vorgehen einer Userin/eines Users wiederum zu verstehen und mit Bedeutung zu füllen werden die Transkripte in der Triangulation herangezogen.

\section{Illustration an zwei Beispielen}

Mirko startet mit den Seiten, die für ihn wichtig sind und eine Bedeutung haben. Wir gehen in dem Moment ins Netz, als er nicht mehr in der Lage ist, mir die Seiten zu Sendungen zu beschreiben, die er regelmässig besucht. "Aah, wie beschreib' ich denn das (4) Pass auf ich hab (..) nämlich ne Internetseite» (Mirko). Hier zeigt sich deutlich, dass das gemeinsame Surfen im Internet für das Verstehen des nicht mehr Beschreibbaren oder sprachlich Fassbaren von immenser Bedeutung ist. Surfwege und Webseiten lassen sich verständlicher visualisieren als nur mündlich beschreiben. Er präsentiert als erstes Internetauftritte, die für ihn zum Zeitpunkt der Absetzung des Senders VIVA Zwei eine besondere Relevanz gewonnen haben. "Es gibt nämlich auch so richtige Initial Initiativen hat's gegeben und da gibt's halt mehrere Internetseiten, wo dann halt so äh an VIVA Zwei gedacht wird.» (Mirko). Diese Webauftritte sind keine offiziellen VIVA Zwei Seiten, sondern private von Fans des ehemaligen Senders ins Leben gerufene Präsenzen, die noch zum Zeitpunkt des Interviews betrieben werden. Mit dem Vorführen dieser Seiten präsentiert sich Mirko mir gegenüber als Wissender im Umgang mit dem Medium Internet. Seine Recherchen und sein Informations- bzw. Kommunikationsbedürfnis im Zusammenhang mit der Programmeinstellung von VIVA Zwei, lenkten seinen Weg auf Seiten, die ihn in eine Gemeinschaft von Gleichgesinnten führte. Diese Seiten werden von «Widerständlern» betrieben, die sich nicht ohne weiteres mit der Einstellung des Senders abfinden wollen. Schon allein die Begrifflichkeiten insbesondere bei «http://www.vivaplus.net», die auch Mirko gebraucht, «und dann finde ich auch ganz cool (..) gibt's hier z. B. ne VIVA Zwei resistance (...) die heisst die älteste Widerstandsseite rund um VIVA Zwei und so» (Mirko), entspringen einer «Protestkultur» und damit dem («aktiven») Widerstand. ${ }^{4}$ Der Protest auf den offiziellen - umgedeuteten - VIVAplus Kommunikationsforen verlagert sich in Foren auf Internetseiten, die dem Sender VIVA Zwei nachempfunden sind. VIVA Zwei wird damit «am Leben erhalten» und ist vom Fernsehen in ein anderes Medium (Internet) verschoben und damit in der Wiederholung rekontextualisiert worden. Dies zeugt von einer Handlungsfähigkeit im Umgang mit den verschiedenen Medien, bei der sich die Userinnen und User bis zu einem gewissen Grad von den Intentionen des Senders befreien, indem sie erst einmal unabhängig von der Wirksamkeit ihres

4 Dieser beginnt mit dem Ins-Leben-Rufen der Websites und Foren, auf denen weiter über VIVA Zwei kommuniziert wird. Letztlich äussert er sich in einem Demonstrationsaufruf in den Foren anlässlich der Umgestaltung des Senders VIVA Zwei zu VIVAplus und endet in der Durchführung der Demonstration vor dem Gebäude des Senders. Hier findet eine Protest-Verlagerung von online (den Foren) nach offline (auf die Strasse) statt. 
Protestes sich das Medium ihren Belangen entsprechend zu Nutze machen. Damit unterlaufen sie die Veränderung (bzw. Auslöschung) von VIVA Zwei zugunsten von VIVAplus, indem sie VIVA Zwei «nicht sterben lassen». Sie inszenieren damit ihren Protest nicht nur in vereinzelten verbalen Attacken auf der offiziellen Seite des neuen Senders, sondern sie versuchen mittels eigener Plattformen, Proteste zu bündeln und diese für ihre Zwecke (gegen den Sender) einzusetzen.

In den Foren dieser Auftritte findet Mirko den Austausch mit Zuschauerinnen und Zuschauern, die wie er die Senderumstellung von VIVA Zwei zu VIVAplus zutiefst bedauern und sich davon übergangen und nicht berücksichtigt fühlen, wie er es im Interview beschreibt. In den Foren findet Mirko den Austausch mit Zuschauerlnnen, die wie er diese Senderumstellung zutiefst bedauern und sich davon übergangen und nicht berücksichtigt fühlen. Gleichzeitig verstärkt der kommunikative Austausch sein Gefühl, gemeinsam etwas bewegen zu können. Am Beispiel der Sendung Zwobot, die er als eine "Art Comedysendung mit so (..) Puppen, aber jetzt nicht besonders also das war jetzt mit also teilweise mit so Socken gemacht also ganz primitiv (..) aber es war halt eher so, es war son bisschen mehr so Medienkritik so Zeitkritik aber so als als Puppenspiel gemacht» (Mirko) beschreibt, exemplifiziert er eine Situation, in der er versuchen will, den Sender zur Wideraufnahme dieser Sendung zu bewegen. Damit ich jedoch weiss, von welcher Sendung er spricht, spielt er mir zuvor einen Trailer auf der Seite von shttp://www.vivaplus. net) vor. Für Mirko ist dies eine Sendung mit «künstlerische[m] Anspruch [...] so Augsburger Puppenkiste für Erwachsene» (Mirko) und entspricht seinen Vorstellungen von Originalität, kritischem Humor und Authentizität. Dafür lohnt es sich, sich einzusetzen. "War ne richtig geile Sendung, also ich finde das auch echt schade, dass das kaputt gemacht worden ist also es muss ja noch irgendwo rumliegen die alten Bänder und (..) ich hab' jetzt gerade bei äh VIVAplus im Forum gepostet äh (..) ob da nicht mal irgendwelche Leute auch Interesse hatten äh hätten, dass man diese Sendung mal wieder zeigt.» (Mirko). Er wendet sich mit seinem Vorschlag, diese Sendung wieder aufzunehmen, jedoch nicht direkt an den neuen Sender VIVAplus, sondern versucht zuerst mittels seines Postings im Forum von 〈http://www.vivaplus.net das Interesse anderer VIVA Zwei Fans an der Wiederaufnahme dieser Sendung zu eruieren. "Und nun hoff' ich mal, dass vielleicht ein paar Leute, die da das Forum besuchen jetzt also von den Usern dann äh (..) ein bisschen Druck machen (..) ja das ist einfach schon ein Vorteil von von diesem äh interaktiven Medien, dass dann eventuell sag' ich mal durch en Druck der Masse oder was (..) Sachen verändert werden können also es hat sich auch schon einiges verändert da bei VIVAplus.» (Mirko). Es wird deutlich, dass Mirko den Vorteil der interaktiven Medien bzgl. dieser Situation in der Mobilisierung von Massen sieht. Ihm ist durchaus bewusst, dass er als einzelner eine viel geringere Chance hat, den Sender zur Wiederaufnahme zu bewegen als dies vereint in einem Verbund von Userinnen bzw. User (und Zuschauerinnen bzw. Zuschauer) zu fordern. Mirko wählt 
hier den Weg, über die Forenkommunikation Gleichgesinnte zu mobilisieren und in einem gemeinsamen Akt auf den Sender Druck auszuüben, um somit Einfluss zu nehmen und den Sender zum Handeln zu bewegen.

Im weiteren Verlauf seines Surfweges durch das Internet zeigt er mir auf der Seite von «http://www.vivaplus.net noch andere die Sendungen, die er früher auf VIVA Zwei besonders geschätzt hat. Der grösste Teil unserer Internetsitzung widmet sich den Seiten, die mit der Absetzung von VIVA Zwei in Verbindung stehen. Danach zeigt er mir zum Vergleich andere Seiten, wie die zur Harald Schmidt Show, deren Angebote er ebenso schätzt. "Ja, also da gehe ich halt auch ab und zu mal rauf (..) und dann guck' ich so, was da so ist ähm (...) weiss nicht da schreiben die dann immer so, da kann man dann so (..) immer lesen, was dann so die Zuschauer, die schreiben ja manchmal so Sprüche für Schmidt, das ist irgend so eine Rubrik (..) und dann kann man gucken, was denn so die Sprüche des Tages waren oder (..) ja oder man guckt, wer als Gast abends da ist oder was weiss ich oder halt einfach man guckt im Archiv so nach, was denn sol was er im Archiv so hal die ham die so einige paar Sachen so als ähm (..) als äh als als als Quicktimemovie da zum zum Anl Angucken da im Archiv.» (Mirko). Am Beispiel der Harald Schmidt Show wird deutlich, dass ihn das Angebot um die Sendung interessiert. Zum einen können dies Informationen sein, aber auch Äusserungen von Zuschauerinnen und Zuschauern wie die "Sprüche für Schmidt», Hinweise auf die Gäste oder das Archiv, in dem er sich Szenen aus vergangenen Sendungen anschauen kann. Die Angebote der Internetpräsenz nutzt er zur Vertiefung und für weiterführende Hintergrundinformationen, die er über das Fernsehen nicht erhalten kann. Hier kann er sich Interessantes noch einmal ansehen «als Quicktimemovie», sich Zusendungen von Zuschauerinnen und Zuschauern durchlesen, die nicht im Fernsehen präsentiert wurden oder auch nur die Gästeliste angucken, um schon im Voraus zu wissen, wer bei Harald Schmidt in der nächsten Sendung eingeladen ist.

Erst zuletzt geht er auf die offiziellen Senderseiten von VIVAplus und VIVA. Diese Seiten besucht er so gut wie nie, wie im Gespräch deutlich wird. Das einzige Interesse liegt hier auf der Sendung Fast Forward mit Charlotte Roche, die er zum Zeitpunkt des Interviews noch schaut, welche aber auf den Sender VIVA verschoben wurde. Zuvor lief die Sendung auf VIVA Zwei, mittlerweile ist sie abgesetzt worden.

Von allen besuchten Webseiten habe ich Screenshots erstellt, um die chronologische Reihenfolge des Surfweges festzuhalten. Auf dieser Grundlage und um das Verhältnis und die Bedeutung der einzelnen Seiten im gesamten Surfweg zu rekonstruieren, entwickelte ich schliesslich das Surfgramm zu Mirkos Onlineaktivitäten. 


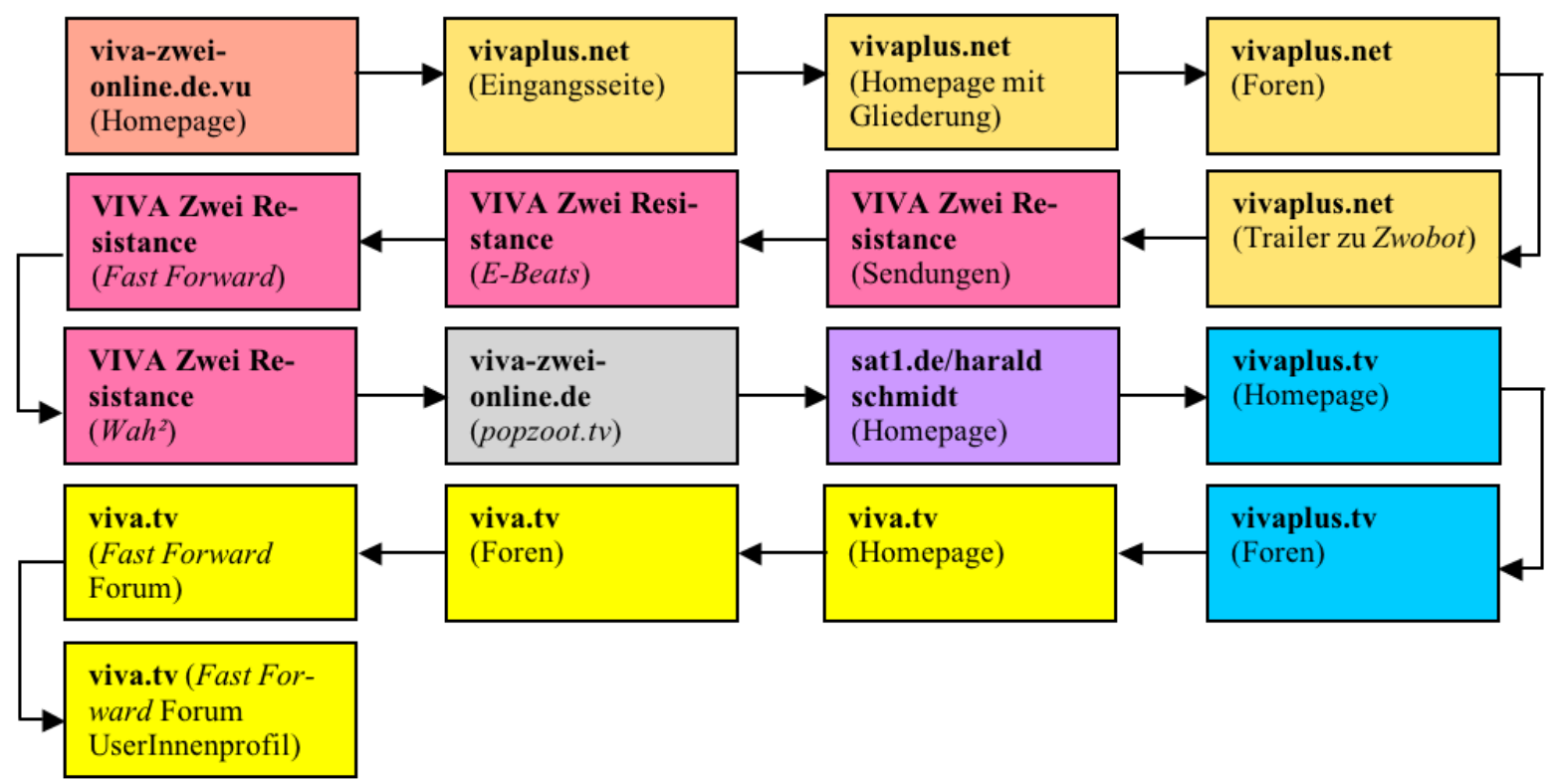

Abbildung 2: Mirkos Surfweg als Surfgramm

Das Surfgramm visualisiert Mirkos Bewegung durchs Netz. Mehr als die Hälfte der Seiten, die wir gemeinsam im Netz besuchen, es sind 10, wie sich im Surfgramm zeigt, beziehen sich auf den Konflikt, den Mirko mit VIVA Zwei und seiner Programmeinstellung austrägt. Er geht hier mit seinen Ausführungen besonders in die Tiefe und macht auf diese Weise seine Verlusterfahrung deutlich. Die letzte dieser Seiten, «http://www.popzoot.tv», präsentiert er mir als ein "Onlineportal für Musikvideos» mit Cliparchiv. Dort sieht er sich mittlerweile die Videos an, die ihn interessieren. Während wir uns auf den Musiksenderauftritten von VIVAplus und VIVA bewegen, wird er das erste Mal im Interview sehr undifferenziert. Er urteilt über diese Seiten eher pauschal und zeigt auf diese Weise seine Ablehnung.

Mirkos Vorgehen im Netz und sein Surfweg zeigen, dass er sich vom für ihn Bedeutsamen zum weniger Wichtigen bewegt. Schon allein die Anzahl von Webseiten, die er aufgrund seiner Enttäuschung durch die Absetzung von VIVA Zwei regelmässig besucht, weisen auf eine hervorgehobene Bedeutung und Präsenz im Internetgebrauch Mirkos zur Zeit des Interviews hin. Er beschäftigt sich ausführlich mit dem Relaunch bei VIVA und der daraus erfolgten Entwicklung und durchforstet das Internet nach Seiten, die für ihn hierfür von besonderer Relevanz sind. Durch die Hinzunahme des Interviews bei der Interpretation wird deutlich, dass er auf der Suche nach Gleichgesinnten im Netz ist, mit denen er Kontakt aufnehmen und sich austauschen möchte. Mirko präsentiert sich hier als ein Nutzer, der weiss, wohin er will und was er vom Internet erhalten kann. Er geht in seinem Sinne selbstbestimmt vor, lässt sich weder durch die Interviewsituation noch durch Verlinkungsstrukturen stark beeinflussen, sondern versucht stets selbst die Kontrolle über die Handlungs- 
situation zu behalten, was sich auch in seiner Forennutzung zeigt. Denn hier sucht er den Austausch und die Kommunikation mit Gleichgesinnten, die ebenso wie er gegen die Senderabsetzung von VIVA Zwei protestieren. Mirko bewegt sich zielgerichtet und wählt Plattformen, die für ihn die relevanten Informationen und Angebote bereitstellen. Seine Suchbewegungen folgen nicht dem Muster des Sich-Treiben-lassens, sondern des Auswahl-treffens. Denn er weiss, auf welche Seiten er gehen muss, um die gewünschten Informationen und Kommunikationsplattformen zu finden und entsprechend dieses Wissens bewegt er sich durch das Netz wie das Surfgramm zeigt. Es wird keine Suchbewegung oder ein Hin- und Herspringen deutlich, sondern er geht intentional vor. Als Orientierungsmassstab dient ihm der Inhalt bzw. der Gegenstand, zu dem er Informationen sucht und Austauschmöglichkeiten finden will. Im Vordergrund stehen für Mirko somit die Suche nach Informationen, der kommunikative Austausch mit Freunden oder themenzentriert wie hier zu VIVA Zwei mit Fremden. Unterstützt wird diese Analyse durch das Aufsuchen der Website zur Harald Schmidt Show, die nicht verlinkt ist mit den zuvor aufgesuchten Seiten. Auch hier verfolgt er ein klares Interesse, welches inhaltlich durch die Interpretation des Interviews gefüllt wird. Genauso verhält es sich mit der Seite zur Sendung von Fast Forward auf der VIVA Website. Auch diese wird nur entsprechend seines Anliegens besucht. Somit lässt sich festhalten, dass mittels des Surfgramms deutlich wird, dass er seinem Interesse folgend nur ganz spezielle Seiten aufsucht, diese jedoch in unterschiedlicher Intensität nutzt, wie die Verweildauer auf den einzelnen Websites zeigt. Das Surfgramm offenbart zum einen die verschiedenen besuchten Seiten innerhalb eines Webauftritts und die entsprechenden Weiterbewegungen, die nicht in dieser Deutlichkeit hervortreten, wenn nur das Interviewtranskript interpretiert würde.

Dies möchte ich noch einmal an einem weiteren Beispiel darstellen und intensivieren:

Als Tamara ins Internet geht, beginnt sie - anders als Mirko - nicht mit den Seiten, die ihr besonders am Herzen liegen, sondern mit Seiten, die ihrem gewöhnlichen, alltäglichen Gebrauch entsprechen. Ihre Startseite ist 〈http://www.freenet.de〉, da sie früher hier ihren E-Mailaccount hatte. Mittlerweile nutzt sie eine Adresse bei 〈http://www.web.de〉, passt jedoch ihre Startseite nicht den neuen Umständen an, denn Interneteinrichtungen überlässt sie ihrem älteren Bruder und arrangiert sich mit dem vorgefundenen Zustand. Das folgende Surfgramm visualisiert Tamaras Surfweg durchs Netz: 


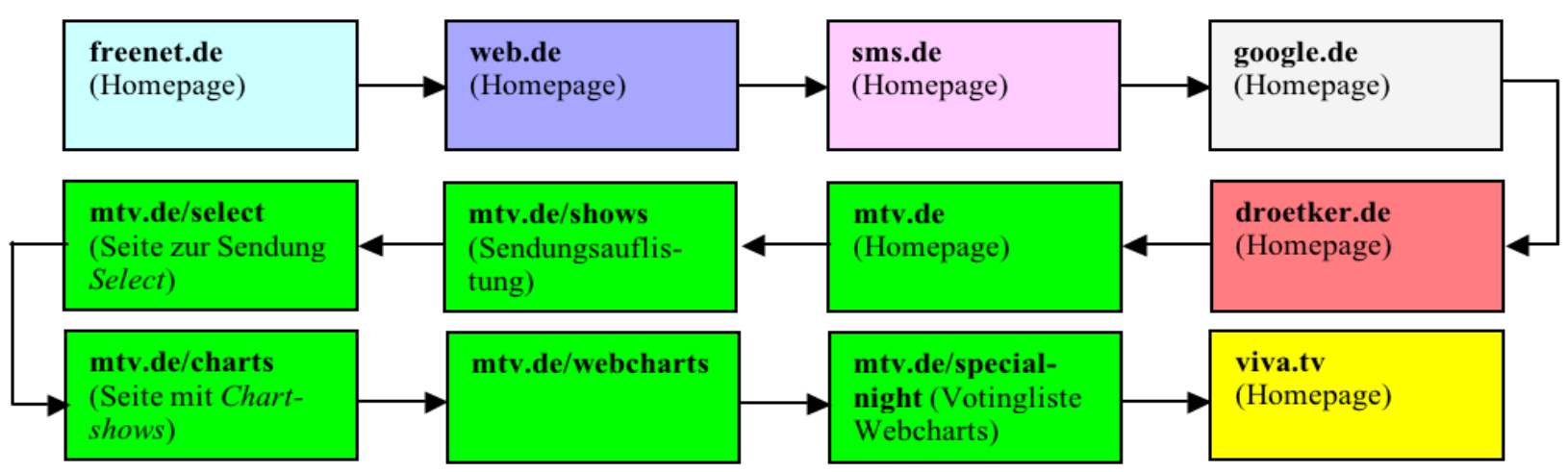

Abbildung 3: Tamaras Surfweg als Surfgramm

Tamaras Surfgramm offenbart, dass sie mit den ersten fünf Seiten einen Überblick über ihren gewöhnlichen Surfweg geben will. Sie zeigt dies anhand der Homepages der einzelnen Websites. "Das ist halt so mein Standard was ich halt so mache als erstes also ich geh eigentlich auf web.de.» (Tamara). Bei «http://www.web. des loggt sie sich ein und erzählt mir, was sie normalerweise auf dieser Seite macht: Zuerst löscht sie die Werbemails und guckt, ob sie neue E-Mails bekommen hat, dann tippt sie Fussballergebnisse, schliesslich beantwortet sie die ihrem Ermessen nach wichtigsten E-Mails. Hier differenziert sie, indem es für sie wichtige E-Mails gibt, die sofort beantwortet werden und unwichtige, die warten können. Nach dem Durchsehen ihres E-Mailaccounts "geh ich meistens zu sms.de» (Tamara). Auf der Website von 〈http://www.sms. de` kann sie jeden Tag eine kostenfreie SMS verschicken. Wenn sie Referate für die Schule zu erstellen hat, besucht sie anschliessend die Homepage von Google. Diese Suchmaschine nutzt sie zur gezielten Recherche von Informationen. Ist sie auf der Suche nach Backrezepten, guckt sie direkt auf die Internetseiten des Webauftritts von Dr. Oetker. «Ja so wenn halt irgendjemand Geburtstag hat oder so und ich Kuchen backen will dann guck ich halt mal so (..) was es da so Schönes gibt an Torten oder so (..) irgendwelche Ideen.» (Tamara). Das WWW ist hier ein Ideengeber für leibliche Genüsse. Auf der Website von Dr. Oetker holt sie sich Rezept- bzw. Backideen und -anregungen. Erst danach geht sie auf die Homepage von MTV. Der Internetauftritt von MTV ist die einzige Präsenz im WWW der vier Musiksender, die sie nutzt. Er ist in doppelter Hinsicht ihre Wunschwebsite, wie aus dem Interview mit Tamara hervorgeht. Zum einen ist es die einzige Präsenz im WWW der vier Musiksender, die sie nutzt, zum zweiten wünscht sie sich hier Musik für die Sendung MTVselect oder für die Webcharts. Anhand der Internetseiten zeigt sie mir, wie das Musiktitel-Voting im Internet funktioniert. Während sie sich auf der MTV Website bis zur Votingliste der Webcharts über Shows, Select und Charts durchklickt, redet sie über die Sendungen, die wir vorher schon besprochen haben. Diese Shows zeigt sie mir beim Netzdurchgang, allerdings nur aufgelistet in der Navigationsleiste zu den Sendungen. Auf meine 
Frage nach dem Zusammenhang von MTV Fernsehen und Internet antwortet sie am Beispiel von Select: "Also ich find es hängt eigentlich ähm MTV insgesamt hängt eigentlich gar nicht damit zusammen halt nur wieder Select die Show (.) weil ähm das halt auch die hat auch immer einen Computer im Studio da stehen und ähm guckt auch selber auf die Homepage und liest auch E-Mails vor direkt und ähm (..) man kann ja auch so Fragen stellen meistens übers Internet und so und ich finde das ist halt so (..) da wird halt auch ziemlich viel reingepackt und die bauen darauf auf.» (Tamara). Interessanterweise beantwortet sie hier meine Frage nach Medienkonvergenz mit der Beschreibung eines intermedialen Phänomens, das auf eine medienkonvergente Handlung zielt. Sie beschreibt konkret das, was sie im Musikfernsehen bei der Sendung Select sieht: das Vorhandensein eines Computers in einer Sendung, auf dem die Internetseite zu der Show selbst aufgerufen ist. In Anlehnung an Rajewsky kann hier von einer Medienkombination gesprochen werden, d.h. es kommt zu einer medialen Präsentation zweier distinkt wahrnehmbarer Medien (vgl. Rajewsky 2002, S. 15). Zuletzt besucht sie die Homepage von VIVA, um mir im Vergleich zu MTV zu erläutern, was ihr an dieser Seite missfällt und warum sie diesen Auftritt in der Regel nicht nutzt.

Zu Tamaras Surfweg im Netz lässt sich festhalten, dass sich bei ihr gewisse Routinen und Umgangspraxen eingestellt haben, denen sie ihrer Gewohnheit folgend nachgeht. Sie bewegt sich vom Gewöhnlichen oder Alltäglichen - ihren Standards, wie sie es selbst nennt - zum Besonderen oder Spezifischen. Das zeigt die Abfolge von aufgesuchten Websites, die sie am Anfang der Sitzung besucht. Sie surft von einer Seite zur nächsten, verweilt dort aber nicht länger. Die Seiten werden quasi je nach Anliegen abgearbeitet. Tamara verweilt nur dann länger, wenn sie etwas Spezielles sucht - wie beispielsweise mit der Suchmaschine google - oder sich inspirieren lassen will - wie bei dem Dr. Oetker Beispiel. Das Spezifische in der Interviewsituation sind die Webseiten des MTV Auftritts. Die ersten fünf Seiten ihres Surfgramms zeigen, dass es ihr in der alltäglichen Nutzung zum einen um Kommunikation und zum zweiten um Information bzw. Informationssuche geht. Doch ist ihr Kommunikationsbedürfnis nicht nur online-online wie bei Mirko ausgeprägt, sondern ebenso online-offline mit dem Schreiben von SMS via Internet auf das Handy. Dieses Inanspruchnehmen kostenfreier SMS weist bzgl. ihrer Handlungsfähigkeit auf Flexibilität im Umgang mit den verschiedenen Medien hin. Sie nutzt das, was sich ihr bietet - SMS lassen sich nicht nur vom Handy aus schreiben. Die spezifische Nutzung der MTV Seiten unterliegt ebenfalls der Suche nach Informationen und darüber hinaus dem Bedürfnis nach Partizipation, was sich in der Teilnahme an Votings offenbart. Ihr scheint es dabei um die Möglichkeit der Partizipation zu gehen, denn hierüber kann sie sich ausdrücken und bestenfalls Einfluss ausüben. Es geht um die Möglichkeit der Einflussnahme durch Partizipation, um die Möglichkeit der Mitbestimmung durch das Äussern eines Wunsches. Auf den Seiten von MTV verweilt sie die längste Zeit, wie durch das Surfgramm offensichtlich wird, was jedoch 
auch der Interviewsituation geschuldet ist. Die Internetnutzung von Tamara weist auf eine gezielte Vorgehensweise hin, sie lässt sich eher selten von den Angeboten im Netz treiben, eben nur dann, wenn sie sich anregen lassen will.

\section{Resümee}

Zusammenfassend kann nun Folgendes für das Surfgramm festgehalten werden: Das Surfgramm lässt sich als Auswertungsinstrument zur Visualisierung von Surfwegen einsetzen. Auf diese Weise bringt es einen zusätzlichen Erkenntnisgewinn für die Internetnutzung. Es steht für die Veranschaulichung der Webseiten, die im Interview besprochen werden, und für die Konkretisierung des im Interview schwer Beschreibbaren. Die Qualität des Visuellen macht den konkreten Surfweg der Userinnen und User sichtbar, ohne dass es sich dabei um eine Produktanalyse handelt, und bildet in dieser Hinsicht eine Kontrastfolie im Abgleich zum Text.

Mit Hilfe des Surfgramms lassen sich in Korrespondenz mit dem Transkripttext die Interpretationen überprüfen und schärfen. Somit ist es auch ein Instrument zur Validierung ${ }^{5}$ der Forschungsergebnisse im Bereich Onlineforschung. Das Surfgramm macht a) die Schwerpunktsetzung, b) den Einstieg und c) die Gewichtung im Verlauf des Netzweges und die daran geknüpften Handlungsdimensionen der Interviewpartnerinnen und -partner sichtbar. Somit liesse sich das Surfgramm sicherlich auch auf andere (Forschungs-)Fragen anwenden, die sich mit dem konkreten Gebrauch des Internets beschäftigen und beispielsweise nach Veränderungs- und Lernprozessen fragen. Denn im Vergleich der einzelnen Surfgramme, die beispielsweise zu unterschiedlichen Zeitpunkten erstellt werden, liessen sich Entwicklungsprozesse abbilden und mit dem entsprechenden Interviewtext interpretieren.

5 Siehe auch zu «Gütekriterien qualitativer Forschung» und zum Problem der Validierung Steinke 2000. 


\section{Literatur}

Bilandzic, Helena; Trapp, Bettina. «Die Methode des lauten Denkens: Grundlagen des Verfahrens und die Anwendung bei der Untersuchung selektiver Fernsehnutzung bei Jugendlichen.»In: Paus-Haase, Ingrid; Schorb, Bernd (Hrsg.). Qualitative Kinder- und Jugendmedienforschung. Theorie und Methoden: ein Arbeitsbuch. München: KoPäd, 2000. 183-209.

Bohnsack, Ralf. Rekonstruktive Sozialforschung: Einführung in Methodologie und Praxis qualitativer Forschung. 4. Auflage. Opladen: Leske und Budrich, 2000.

Fabel-Lamla, Melanie; Tiefel, Sandra. «Fallrekonstruktion in Forschung und Praxis - Einführung in den Themenschwerpunkt.» Zeitschrift für qualitative Bildungs-, Beratungs- und Sozialforschung 2 (2003): 189-198.

Fischer-Rosenthal, Wolfram; Rosenthal, Gabriele. «Warum Biographieforschung und wie man sie macht.» Zeitschrift für Sozialisationsforschung und Erziehungssoziologie, 17. Jg., Heft 4 (1997): 405-427.

Flick, Uwe. Triangulation: Eine Einführung. Wiesbaden: VS Verlag, 2004.

Marotzki, Winfried. «Forschungsmethoden der erziehungswissenschaftlichen Biographieforschung.» In: Krüger, Heinz-Hermann; Marotzki, Winfried (Hrsg.). Erziehungswissenschaftliche Biographieforschung. 2. Auflage. Opladen: Leske und Budrich, 1995. 55-89.

Marotzki, Winfried. «Neue kulturelle Vergewisserungen: Bildungstheoretische Perspektiven des Internet.» In: Sandbothe, Mike; Marotzki, Winfried (Hrsg.). Subjektivität und Öffentlichkeit: Kulturwissenschaftliche Grundlagenprobleme virtueller Welten. Köln: Herbert von Harlem Verlag, 2000. 236-258.

Marotzki, Winfried. «Online-Ethnographie - Wege und Ergebnisse zur Forschung im Kulturraum Internet.» In: Bachmair, Ben; Diepold, Peter; Witt, Claudia de (Hrsg.). Jahrbuch Medienpädagogik 3. Opladen: Leske und Budrich, 2003. 149-165.

Merton, Robert; Kendall Patricia. «Das fokussierte Interview.» In: Hopf, Christel; Weingarten, Elmar (Hrsg.). Qualitative Sozialforschung. Stuttgart: Klett-Cotta, 1979. 171-204.

Paus-Haase, Ingrid. «Medienrezeptionsforschung mit Kindern - Prämissen und Vorgehensweisen: Das Modell der Triangulation.» In: Paus-Haase, Ingrid; Schorb, Bernd (Hrsg.). Qualitative Kinder- und Jugendmedienforschung. Theorien und Methoden: ein Arbeitsbuch. München: KoPäd, 2000: 15-32.

Schütze, Fritz. "Biographieforschung und narratives Interview.» Neue Praxis 3 (1983): 283-293.

Rajewsky, Irina O. Intermedialität. Tübingen und Basel: Francke Verlag, 2002.

Steinke, Ines. "Gütekriterien qualitativer Forschung.»In: Flick, Uwe; Kardorff, Ernst von; Steinke, Ines (Hrsg.). Qualitative Forschung: Ein Handbuch. Reinbek bei Hamburg: Rowohlt Taschebuch Verlag, 2000: 319-331. 